\title{
A New Kind of Position Location Sensor Based on Encode and the Application Research
}

\author{
Junxing Zhang ${ }^{1}$ and Chunjuan Bo ${ }^{*}, 2$ \\ ${ }^{I}$ College of Information and Communication Engineering, Dalian Nationalities University, Dalian, Liaoning, 116600, \\ P.R. China \\ ${ }^{2}$ College of Electromechanical Engineering, Dalian Nationalities University, Dalian, Liaoning, 116600, P.R. China
}

\begin{abstract}
This paper proposed a plane position detection method based on coding identification, discusses key problems to be solved in design of interactive electronic whiteboard with this method, gives structure and optical path design method of pen-based sensing system, discusses plane position coding method, comes up with solutions of coding of discontinuous code area and incremental coding of continuous code area, and analyzes the realization and performance. As for deviation in design of interactive electronic whiteboard with this method, three-point calibration algorithm is used for the system. This method effectively solves deviation caused by panning, zooming and rotation between image and whiteboard. The electronic whiteboard designed with this method is characterized by low cost, simple structure, etc.
\end{abstract}

Keywords: Calibration algorithm, interactive electronic whiteboard, optical identification of coding, position coding, position detection.

\section{INTRODUCTION}

Interactive electronic whiteboard is a multimedia tool for teaching, training, meeting, demonstration, etc. that came into being with the development of multimedia technology, computer technology and display technology in the late 1990s. It uses special digital whiteboard to sense position of electronic pen that moves on it, and transmits it to the computer. After software processing, the motion track of pen is displayed on the whiteboard with projector to finish writing and other functions. Endowing the traditional multimedia system with interactive function, electronic whiteboard is deemed to be a new teaching tool to replace traditional blackboard. According to the detection method used, electronic whiteboard falls into scanning, touch, laser positioning and wireless positioning, etc. [1]. At present, electronic whiteboard is not popular due to expensive patents and products related to it.

Drawing on the working principle of optical mouse, this paper puts forward a new position detection method based on position coding identification, and applies it to design of electronic whiteboard system.

The optical mouse receives a series of images from the motion track with built-in CMOS camera, and analyzes change in position of characteristic points on these images with corresponding software to judge direction and distance of motion of mouse to position the optical mouse. The optical mouse is capable of determining direction and distance of motion, but it can only detect relative amount of motion without detecting absolute position.

*Address correspondence to this author at the College of Electromechanical Engineering, Dalian Nationalities University, Dalian, Liaoning, 116600, P.R. China; Tel: +86 13609868793; E-mail: bcj@dlnu.edu.cn

1874-4443/15
Therefore, we set coding information on the electronic whiteboard or projection screen, collect a series of images of motion track through CMOS image sensor in electronic pen that moves on it, analyze coding information in the image to determine coordinates of electronic pen, and transmit the information to computer in a wireless way to finish functions of interactive electronic whiteboard.

\section{STRUCTURAL DESIGN OF PEN-BASED PHOTOELECTRICAL DETECTING SYSTEM}

The structural design of detecting system draws on design concept of pen-based optical mouse which is installed with CMOS image sensor to collect motion images and analyze the characteristic information $[2,3]$. We make the detecting system like a pen. The CMOS mage sensor in penbased detecting system (hereinafter referred to electronic pen) collects image information of electronic whiteboard or projection screen near the motion track of electronic pen, extracts corresponding coding information through image processing to determine position of electronic pen, and transmits the information of position to computer.

The structure of electronic pen is shown in Fig. (1). It is mainly composed of light source, image acquisition light path, CMOS image sensor and image processing system, wireless communication system, key, etc.

Light source system: high-power diode is used as point light source. Infrared diode can be used as needed, which has no impact on human vision. Other colors can also be considered, such as blue light-emitting diode.

Image acquisition light path: the working face below motion track of electronic pen is lit up by light source, while image acquisition light path transmits image information to the image sensor. The structure is shown in Fig. (2). It is mainly composed of camera lens, lens, reflector, etc. The 
effective area of image in electronic pen varies with writing posture to a certain degree, but the minimum effective area is above $1.4 \mathrm{~cm} \times 1.2 \mathrm{~cm}$.

Image sensor: CMOS process image sensor is used, the advantage of which is directly generating digital signal of image. Image sensor with light-sensing area of $1 / 8^{\mathrm{n}}$, resolution of $640 \times 480$ and frame rate of $60 \mathrm{fps}$ is used in this system.

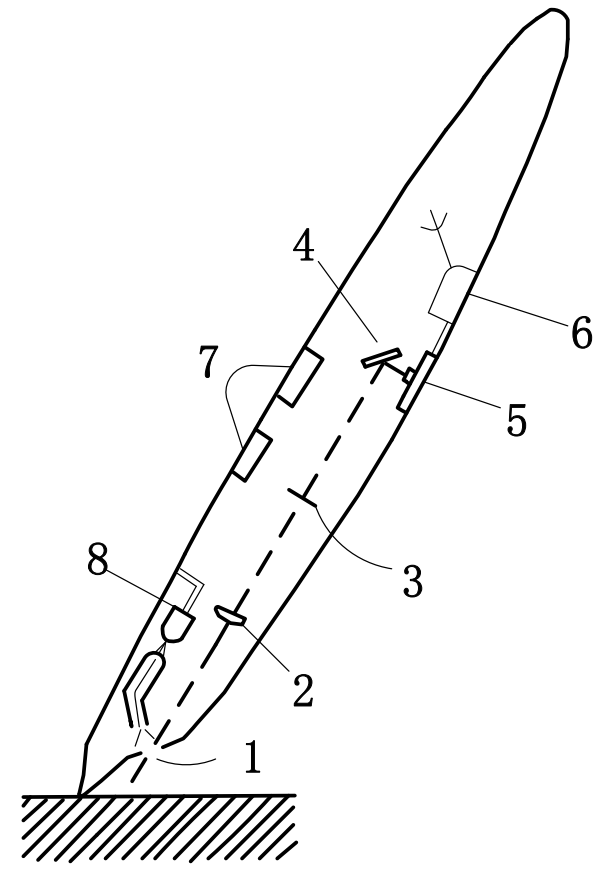

1. Lighting hole

2、3、4 .Image acquisition light path

5. CMOS sensor

6. Processor and communication system

7. Key

8. Light source system

Fig. (1). Structure of electronic pen.

Image processing system: image processing and coding identification is finished with certain algorithm. Image processing chip integrated with CMOS image sensor, or separate image processing chip can be used in design. Chip containing large program and data space should be used in model selection to reduce volume of system. In order to further reduce volume to facilitate installation, $\mathrm{BGA}$ or $\mathrm{COB}$ technology can be used for packaging.

Wireless communication system: transmit position and other information to computer. Bluetooth is a good choice since the communication distance is not very long.

Key: two keys are set, simulating left and right key of mouse.

\section{POSITION CODING ALGORITHM}

Detection of plane position is to determine $x$ and $y$ coordinates with vertical and horizontal coding. The coding is two-dimensional coding, while that of digital level is onedimensional coding. In coding of plane position, we put forward two coding methods: coding of discontinuous code area and incremental coding [4].

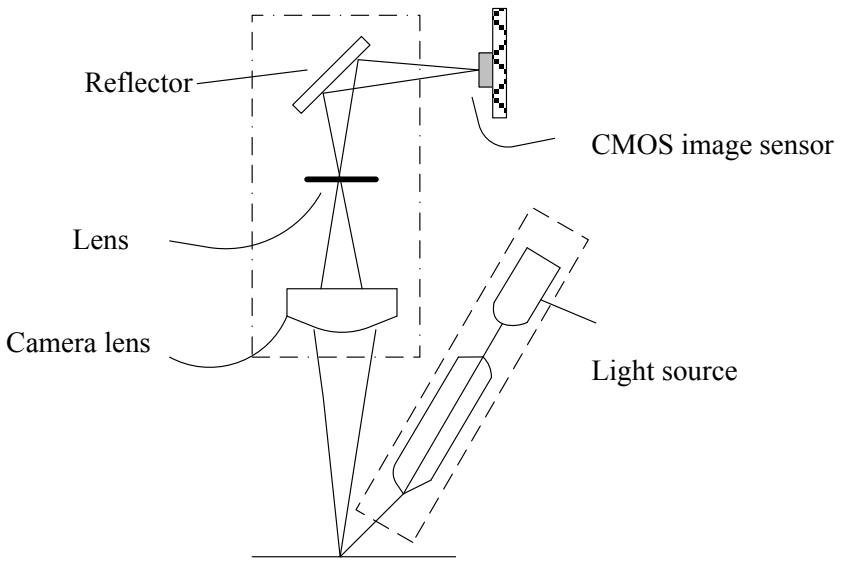

Fig. (2). Structure of light path of system.

In order to make for description of coding algorithm, several terms are defined:

Bar code (code element): basic unit of coding. Its width $d$ is equal in this system and is in black/white (or grey/white).

Kind of bar code $\mathrm{k}$ : for bar codes with a width of $\mathrm{d}$, different type of bar codes can be formed by adjusting proportion of width of two colors. The total number of types of bar code is called kind, $\mathrm{k}$.

Code area: obtain any section of image from continuous coding. This section of image is different from other sections of image of the same length. The smallest section of image that meets this condition is called code area.

Code value of code area: calculate the value of bar codes in the code area according to type and weight. The sum of values is code value of code area.

\subsection{Coding of Discontinuous Code Area}

For centrifugal extruder, the great difference from conventional screw extruder is the theory of solid-plug conveying. The solid-plug conveying of conventional screw extruder is based on the friction between the material and the barrel, or the screw, whereas the solid-plug conveying of centrifugal extruder is based on the centrifugal force field. In this paragraph, the solid-plug conveying theory of centrifugal extruder is studied by the mathematical analysis.

Suppose that width of bar code is D, kind is $\mathrm{K}$, each code area contains $m$ bar codes, and total number of code bars in one direction is $N$. If the condition below is true:

$k^{m} \geq N-m+1$

By adjusting the order of bar codes, there must be a sequence of code bars: $f_{1}, f_{2}, f_{3}, f_{4}, \ldots, f_{N}$. For any code area containing $m$ bar codes $M_{i}: f_{i+1}, f_{i+2}, \ldots, f_{i+m}$, which is shown in Table 1, the kind and arrangement of bar codes is different from that of all other code areas (i.e. different code value of code area). The coding sequence can be obtained through corresponding computer program. 
Table 1. Code area and coding order.

\begin{tabular}{|c|c|}
\hline Number of code area & Order of bar codes in code area \\
\hline \hline 0 & $f_{1}, f_{2}, \ldots, f_{m}$ \\
\hline 1 & $f_{2}, f_{3}, \ldots, f_{m+1}$ \\
\hline$\vdots$ & $\vdots$ \\
\hline $\mathrm{N}-\mathrm{m}$ & $f_{N-m+1}, f_{N-m+2}, \ldots, f_{\mathrm{N}}$ \\
\hline
\end{tabular}

For instance, when $\mathrm{k}=2, \mathrm{~m}=4$, based on formula (1),

$$
\begin{aligned}
N \leq & k^{m}+m-1 \\
& \leq 2^{4}+3=19
\end{aligned}
$$

Table 2. Coding, code area and code value.

\begin{tabular}{|c|c|c|}
\hline Code area & Code bar of code area & Code value of code area \\
\hline \hline 0 & 0000 & 0 \\
\hline 1 & 0001 & 1 \\
\hline 2 & 0011 & 3 \\
\hline 3 & 0111 & 7 \\
\hline 4 & 1111 & 15 \\
\hline 5 & 1110 & 14 \\
\hline 6 & 1101 & 13 \\
\hline 7 & 1011 & 11 \\
\hline 8 & 0110 & 6 \\
\hline 9 & 1100 & 12 \\
\hline 10 & 1001 & 9 \\
\hline 11 & 0010 & 2 \\
\hline 12 & 0101 & 5 \\
\hline 13 & 1010 & 10 \\
\hline 14 & 0100 & 4 \\
\hline 15 & 1000 & 8 \\
\hline Coding & 0000111101100101000 \\
\hline \multicolumn{2}{|c|}{} \\
\hline
\end{tabular}

There is a coding sequence containing 19 code bars. Obtain a code area containing 4 code bars from this sequence, this code area is unique (the category or order of code bars is different from that of other code areas, i.e. code value of code area is different). The coding sequence can be expressed as: 0000111101100101000. The number of code area, order of code bars in the code area and code value of code area is shown in Table 2.

The coding is shown in Fig. (3).

As number of code area does not follow the order of code values in code area, this coding method is called coding of discontinuous code area.

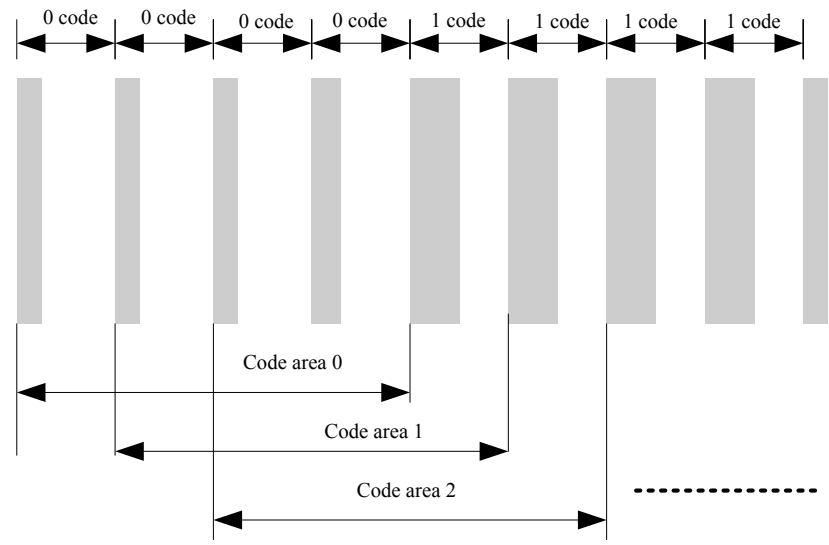

Fig. (3). Coding example.

\subsection{Incremental Coding of Continuous Code Area}

Incremental coding of continuous code area follows the incremental order of code values in the code area. Generally, number of code area equals the code value. In this coding method, each code area is composed of start code bar and coding code bar. Start code bar indicates start of code area, and coding code bar differentiates different code area.

Suppose the kind of bar code is $\mathrm{K}$, each code area contains $\mathrm{m}$ bar codes, and both $\mathrm{K}$ and $\mathrm{m}$ contain start code, the total number $\mathrm{S}$ of code areas with different code values can be:

$(k-1)^{m-1}$. Suppose that $\mathrm{k}=4$, the structure of code bar is shown in Fig. (4).

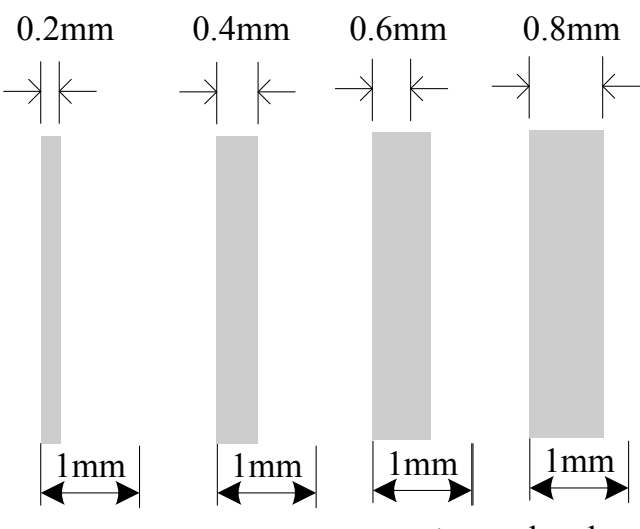

a code c code

d code

Fig. (4). Structure of bar code.

where, a code is start code, and b, c, d are code bars expressed as 0,1 and 2 . Incremental coding is used for arrangement of code areas in system design. Suppose that $\mathrm{m}=4$, and start code is not considered in calculation of code value of code area, code value $M$ of any code area can be:

$M=k_{1} \times 3^{0}+k_{2} \times 3^{1}+k_{3} \times 3^{2}$ 
Coefficients $k_{1}, k_{2}$ and $k_{2}$ are kinds of code bars at corresponding position. Number of code areas, arrangement of code bars and corresponding code values are shown in Table 3.

In order to completely identify each code area, the acquisition window must contain 4 complete bar codes. It can be known from the order of arrangement that the four bar codes must contain a start code. As incremental coding is used, the value of code of the next code area is always 1 larger than that of the last one. Therefore, the number of current code area can be determined according to start code and 3 bar codes on both sides in the code area. In Fig. (5), it is easy to calculate the number of code area of start code: 2010 , i.e. code area 57, and the number of last code area is 2002 , i.e. code area 56.

Table 3. Incremental coding.

\begin{tabular}{|c|c|c|c|c|c|}
\hline Code area & Code bar 1 & $\begin{array}{c}\text { Code bar 3 } \\
\text { K3 }\end{array}$ & $\begin{array}{c}\text { Code bar 4 } \\
\text { K2 }\end{array}$ & $\begin{array}{c}\text { Code bar 5 } \\
\text { K1 }\end{array}$ & $\begin{array}{c}\text { Code Value } \\
\text { of code area }\end{array}$ \\
\hline \hline Code area 0 & $\mathrm{a}$ & 0 & 0 & 0 & 0 \\
\hline Code area 1 & $\mathrm{a}$ & 0 & 0 & 1 & 1 \\
\hline Code area 2 & $\mathrm{a}$ & 0 & 0 & 2 & 2 \\
\hline Code area 3 & $\mathrm{a}$ & 0 & 1 & 0 & 3 \\
\hline Code area 4 & $\mathrm{a}$ & 0 & 1 & 1 & 4 \\
\hline$\vdots$ & $\vdots$ & $\vdots$ & $\vdots$ & $\vdots$ & $\vdots$ \\
\hline Code area 25 & $\mathrm{a}$ & 2 & 2 & 1 & 25 \\
\hline Code area 26 & $\mathrm{a}$ & 2 & 2 & 2 & 26 \\
\hline
\end{tabular}

\section{METHOD FOR COORDINATE TRANSFOR- MATION AND SYSTEM CALIBRATION}

In practical application, due to installation and regulation of projector and other factors, the image of projector is not completely consistent with projection screen or electronic whiteboard (as shown in Fig. 5). The correspondence between screen or electronic whiteboard and projected image should be first solved.

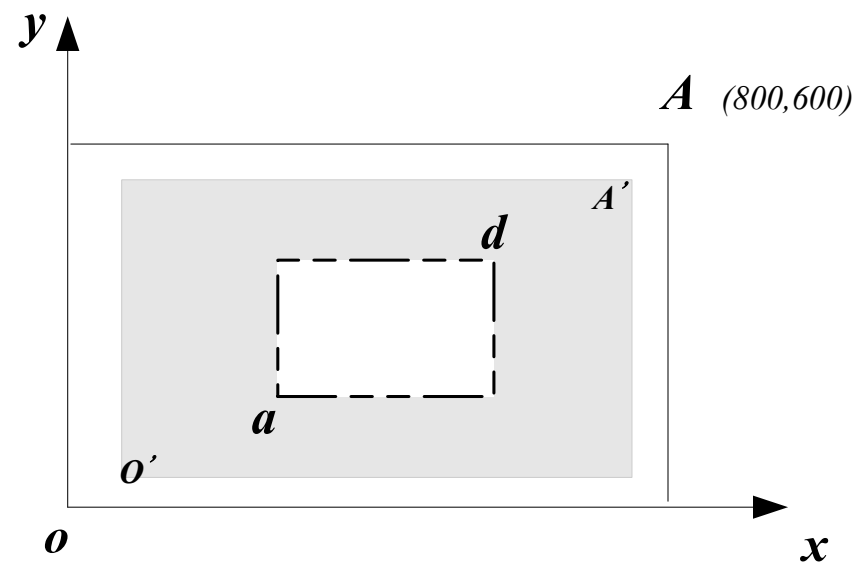

Fig. (5). Diagram of system calibration geometry.
The traditional 2-point correction algorithm only considers error due to panning and zooming between image and whiteboard without taking into account deviation caused by migration at certain angle between projected image and whiteboard $[5,6]$ (as shown in Fig. 6).

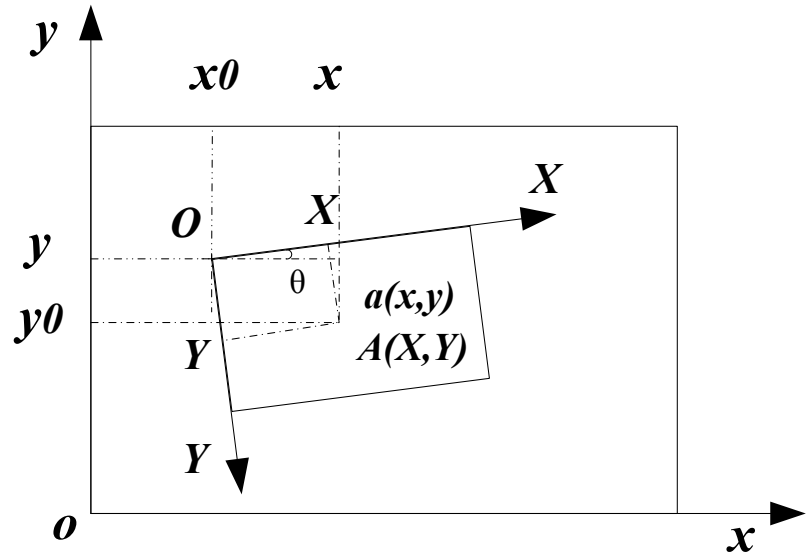

Fig. (6). Diagram of coordinate system geometry.

In order to find a correction algorithm to effectively eliminate the deviation for the above three reasons, we draw on the correction method of touch screen [7,8]. Suppose that there is a point corresponding to any point in logical coordinate system in the physical coordinate system. Any point in logical coordinate system is expressed with vector $D$, and the point corresponding to it in physical coordinate system is expressed with vector $D_{p}$, which is shown in Fig. (7), then $D_{p}=M D$, where $D$ refers to any point in logical coordinate system, and $D_{p}$ refers to the point corresponding to $D$ in physical coordinate system. $\mathrm{M}$ is conversion matrix, then:

$$
\begin{aligned}
D_{p}= & {\left[X_{p}, Y_{p}\right] } \\
& =\left[R_{p} \cos \theta_{p}, R_{p} \sin \theta_{p}\right] \\
D= & {[X, Y] } \\
& =[R \cos \theta, R \sin \theta]
\end{aligned}
$$

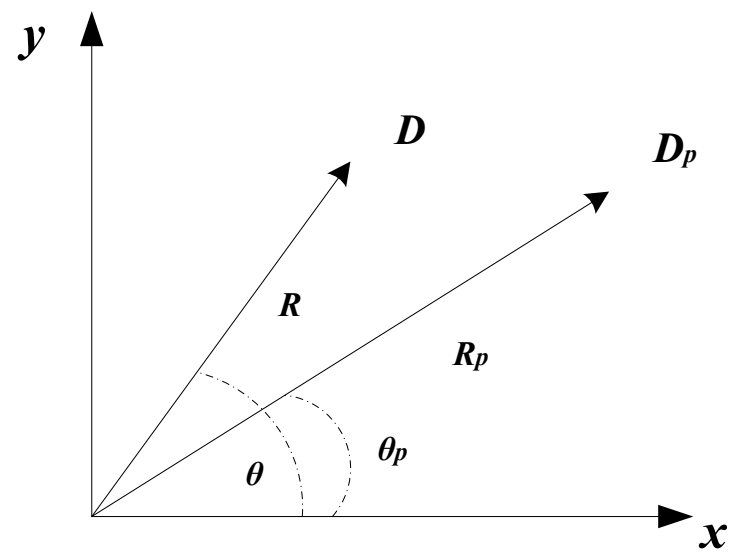

Fig. (7). Vector representation of point in coordinate system. 
Considering the causes of the above three kinds of deviation, the process of coordinate transformation can be described as the combination of three basic geometric transformations: rotation, zooming and panning.

First, consider angular deviation (rotation): before calibration, suppose that angular deviation between whiteboard and image $\theta_{r}=\theta_{D}-\theta$, the following can be derived from formula (5):

$$
\begin{aligned}
D^{\prime}= & {[X, Y] } \\
& =\left[R \cos \left(\theta+\theta_{r}\right), R \sin \left(\theta+\theta_{r}\right]\right.
\end{aligned}
$$

Secondly, consider deviation caused by image zooming, formula (6) can be further expressed as:

$$
\begin{aligned}
D^{\prime \prime}= & {[X, Y] } \\
& =\left[K_{x} R \cos \left(\theta+\theta_{r}\right), K_{y} R \sin \left(\theta+\theta_{r}\right]\right.
\end{aligned}
$$

where, $K_{x}$ is zooming coefficient in $x$ direction, and $K_{y}$ is zooming coefficient in $y$ direction.

Finally, consider deviation caused by panning, $D_{p}$ can be expressed as:

$$
\begin{aligned}
D_{p}= & {[X, Y] } \\
& =\left[K_{x} R \cos \left(\theta+\theta_{r}\right)+X_{T}, K_{y} R \sin \left(\theta+\theta_{r}\right)+Y_{T}\right]
\end{aligned}
$$

where, $X_{T}$ is panning in $x$ direction, and $Y_{T}$ is panning in $y$ direction.

When angular deviation $\theta_{r}$ is relatively small, approximately $\sin \theta_{r} \approx \theta_{r}, \cos \theta_{r} \approx 1.0$, thus:

$$
\left\{\begin{array}{l}
\sin \left(\theta+\theta_{r}\right) \approx \sin \theta+\theta_{r} \cos \theta \\
\cos \left(\theta+\theta_{r}\right) \approx \cos \theta-\theta_{r} \sin \theta
\end{array}\right.
$$

Then:

$$
\begin{aligned}
D_{p}= & {[X, Y] } \\
& =\left[K_{x} R \cos \theta-\theta_{r} K_{X} R \sin \theta+X_{T}, K_{y} R \sin \theta+\theta_{r} K_{y} R \cos \theta+Y_{T}\right] \\
& =\left[K_{x} X-\theta_{r} K_{x} Y+X_{T}, K_{y} Y+\theta_{r} K_{y} X+Y_{T}\right]
\end{aligned}
$$

Formula (10) can be further expressed as:

$$
\left\{\begin{array}{l}
X_{p}=A X+B Y+C \\
Y_{p}=D X+E Y+F
\end{array}\right.
$$

There are 6 undetermined coefficients in the above formula: $A, B, C, D, E$ and $F$. The system can solve these coefficients with only three correction points. Suppose that in the projected image, spacing distance is long enough, logical coordinates are $\left(x_{0}, y_{0}\right),\left(x_{1}, y_{1}\right),\left(x_{2}, y_{2}\right)$, and corresponding physical coordinates are $\left(X_{p 0}, Y_{p 0}\right)$. $\left(X_{p 1}, Y_{p 1}\right)$, $\left(X_{p 2}, Y_{p 2}\right)$, the following equations are tenable:

$$
\left\{\begin{array}{l}
X_{p 0}=A x_{0}+B y_{0}+C \\
Y_{p 0}=D x_{0}+E y_{0}+F \\
X_{p 1}=A x_{1}+B y_{1}+C \\
Y_{p 1}=D x_{1}+E y_{1}+F \\
X_{p 2}=A x_{2}+B y_{2}+C \\
Y_{p 2}=D x_{2}+E y_{2}+F
\end{array}\right.
$$

The 6 coefficients can be solved with formula group (12), thus transformation of logical coordinates into physical coordinates can be finished with formula(10).

Deviation caused by panning, zooming and rotation between image and whiteboard is considered in the above three-point correction algorithm.

\section{CONCLUSION}

This paper proposed a method for absolute position detection based on coding and identification of coding, and applies the method to design of electronic whiteboard system. The paper discusses plane position coding method, puts forward solutions of coding of discontinuous code area and incremental coding of continuous code area, and analyzes the realization and performance. As for the deviation in design of interactive electronic whiteboard with this method, three-point calibration algorithm is used for the system. This method effectively solves deviation caused by panning, zooming and rotation between image and whiteboard. The electronic whiteboard designed with this method is characterized by low cost, simple structure, etc. with good prospect for application and popularization.

\section{CONFLICT OF INTEREST}

The author confirms that this article content has no conflict of interest.

\section{ACKNOWLEDGEMENTS}

This work was financially supported by Natural Science Foundation of Liaoning Province (Grant No. 2013020018), General Science Research Projects of Liaoning Provincial Department of Education (Grant No. L2014536), Fundamental Research Funds for Central Universities (Grant No. DC201501010401, DC201501060201).

\section{REFERENCES}

[1] T. Rappaportw, and J. Reed, "Position location using wireless communication on highways of the future", IEEE Communications Magazine, vol. 34, no.10, pp. 33-41, 1996.

[2] R. P. Wood, J. Harcel, A. F. Hou, and S. Plotkin, "Transmitter pen location system", US Patent 6,414,673, October 11, 1998.

[3] H. -Y. Yang, and S. -G. Lee, "Pen type optical mouse device", US Patent 7,098,894, August 29, 2006.

[4] Z. Jinxing, S. lixin, and Z. Jiyin, "Two-dimension position encoded and recognition technology", Computer Science and Information Technology, vol. 1, pp. 370-373, 2009.

[5] L. Jagannathan, and C. V. Jawahar, "Perspective correction methods for camera-cased document analysis", In: Proceedings of CBDAR'05, Seoul, Korea, pp. 148-154, 2005. 
[6] M. Xiao, L. Xiangdong, and X. Wei, "Position measurement method based on linear array CCD with inverting prism", SPIE, vol. 5633, pp. 265-267, 2005.

[7] J. Lin, M. Xing, and D. Sha, "Distortion measurement of CCD imaging system with short focal length and large- field objective",
Optics and Lasers in Engineering, vol. 43, no. 10, pp. 1137-1144, 2005.

[8] C. E. Vadels, How to Calibrate Touch Screens.www.embedded.com.

(C) Zhang and Bo; Licensee Bentham Open.

This is an open access article licensed under the terms of the (https://creativecommons.org/licenses/by/4.0/legalcode), which permits unrestricted, non-commercial use, distribution and reproduction in any medium, provided the work is properly cited. 